\title{
Diastolic Heart Function and Myocardial Electrical Instability in Patients with Q-wave Myocardial Infarction
}

\author{
Ravshanbek D. Kurbanov, PhD, ScD; A. A. Kilichev; \\ Guzal U. Mullabaeva, $\mathrm{PhD}^{*}$ \\ The Republican Specialized Center of Cardiology, Tashkent, Uzbekistan
}

\begin{abstract}
The study included 131 male patients between the ages of 30 and 69 (51.9 9.13 years) with primary Q-wave myocardial infarction (Q-MI). All patients underwent clinical examination, including a physical examination, medical history, ECG in 12 conventional leads, echocardiography, and 24-hour ECG monitoring from the $10^{\text {th }}$ to the $14^{\text {th }}$ day of MI. The progression of left ventricular diastolic dysfunction in Q-MI patients is associated with a longer history of coronary heart disease and arterial hypertension. With worsening diastolic dysfunction, a marked decrease in LV systolic function is revealed. The severe diastolic dysfunction in Q-MI patients is closely associated with myocardial electrical instability. (Int J Biomed. 2015;5(4):192-194.)
\end{abstract}

Key words: diastolic heart function; Q-wave myocardial infarction; 24-hour ECG monitoring; myocardial electrical instability.

\section{Abbreviations}

LVEDD, left ventricular end-diastolic dimension; LVESD, left ventricular end-systolic dimension; LVEDV, left ventricular end-diastolic volume; LVESV, left ventricular end-systolic volume; LAESV, left atrium end-systolic volume; IVST, interventricular septal thickness; LVPWT, left ventricular posterior wall thickness; LVM, left ventricular mass; EF, ejection fraction; PVCs, premature ventricular contractions; VA, ventricular arrhythmias; PE, peak early filling velocity; PA, peak atria1 filling velocity; EPIA, early post-infarction angina.

\section{Introduction}

The main problem of patients with coronary heart disease (CHD), especially after myocardial infarction (MI) and development of left ventricular remodeling, is preventing reinfarction, congestive heart failure, and cardiac arrhythmias[1,2].

The relationship between pro-arrhythmic indicators and systolic dysfunction is well defined, but the role of diastolic dysfunction (DD) in the formation of myocardial electrical instability remains unclear [3]. It is necessary to clarify the role played by formation of the arrhythmogenic mechanisms of ventricular arrhythmias in CHD patients, depending on the degree of left ventricular dysfunction [4].

*Corresponding author: Guzal U. Mullabaeva, PhD. The Republican Specialized Center of Cardiology, Tashkent, Uzbekistan. E-mail: anchomed@gmail.com

\section{Materials and Methods}

The study included 131 male patients between the ages of 30 and 69 (51.9 99.13 years) with primary Q-MI. The study was approved by the Republican Specialized Center of Cardiology Ethics Committee. Written informed consent was obtained from each patient.

The treatment of acute MI was carried out in accordance with recommendations for the Management of Patients with ST-Elevation Myocardial Infarction and included thrombolytic therapy, early administration of beta-blockers, antiplatelet agents, anticoagulants, nitrates, statins, ACE inhibitors, and loop diuretics. All patients underwent clinical examination, including a physical examination, medical history, ECG in 12 conventional leads, echocardiography, and 24-hour ECG monitoring on the $10^{\text {th }}$ through the 14th days of MI.

Echocardiography and Doppler sonography study carried out on the unit «Sonoline Versa Pro» by standard methods using the recommendations of the American Society 
of echocardiography [5]. The following parameters were measured and calculated: IVST, LVPWT, LVEDD, LVESD, LVEF, LVEVD, LVESV, and LVM (LVM was calculated using the formula R.Devereux [6]). Further analysis was indexed to body surface area indicators: LVEDVI, LVESVI, IVSI, LA ESVI, and LVMI.

As an indication, to the greatest extent reflecting the process of cardiac remodeling, RWT was calculated by the formula (IVS+PW)/LVEDD A value of $\geq 0.45$ showed an increase in RWT. The diastolic function was evaluated by Doppler echocardiography. A decrease in PE/PA ratio less than 1.0 was considered as a sign of DD.

To characterize the PVCs, the B. Lown and M. Wolf classification (1971) and the prognostic classification of J.Bigger (1984) were used. Hourly qualitative and quantitative assessment of PVCs was performed in accordance with the Lown-Wolf gradation: Class 0 - absence of PVCs, Class I rare monomorphic PVCs; Class II - frequent single PVCs; Class III - polymorphic (polytopic) PVCs; Class IVA - paired PVCs; Class IVB - group PVCs; Class V - early PVCs, R/T phenomena. After MI, according to the J.Bigger classification, PVCs $>10$ per hour, pair and group PVCs are potentially hazardous ventricular arrhythmias. Follow-up was 24 months.

The obtained data were processed using computer software Microsoft Excel, STATISTICA 6 and Biostat. The mean (M) and standard deviation (SD) were calculated. The odds ratio (OR) and 95\% confidence interval (95\% CI) were calculated using logistic regression. Group comparisons with respect to categorical variables are performed using chi-square tests or, alternatively, Fisher's exact test when expected cell counts were less than 5. Correlations were examined using regression analysis and Spearman rank correlation coefficient. A probability value of $P<0.05$ was considered statistically significant.

\section{Results and Discussion}

To assess the relationship between LV systolic dysfunction and LV diastolic dysfunction, patients were divided into 2 groups (Table 1): a group with preserved LV systolic function $(\mathrm{EF} \geq 50 \%)$ and a group with a reduced $\mathrm{EF}$ $(\mathrm{EF}<50 \%)$. Analysis of the prevalence of different types of LV diastolic dysfunction showed that severe diastolic dysfunction (pseudonormal and restrictive types) was significantly more frequent in patients with reduced LV systolic function $[7,8]$.

Table 1.

Prevalence of $L V$ diastolic dysfunction in patients with different $L V$ contractility

\begin{tabular}{|l|c|c|c|}
\hline Diastolic dysfunction & LV EF $>50 \%$ & LV EF $<50 \%$ & $P$ \\
\hline Impaired relaxation & $36 / 80(45 \%)$ & $44 / 80(55 \%)$ & 0.27 \\
\hline Pseudonormal filling & $15 / 42(35.7 \%)$ & $27 / 42(64.3 \%)$ & 0.016 \\
\hline Restrictive filling & $4 / 25(16 \%)$ & $21 / 25(84 \%)$ & 0.00001 \\
\hline
\end{tabular}

All patients were divided into 3 groups, depending on the severity of LV diastolic dysfunction: Group $1(n=80)$ included patients with impaired relaxation of LV (type 1),
Group $2(n=42)$ included patients with pseudonormal filling (type 2), and Group 3 ( $\mathrm{n}=25)$ included patients with restrictive filling (type 3).

Analysis of clinical and anamnestic indicators (Table 2) showed that patients with severe LV diastolic dysfunction were more likely to have anterior localization of MI $(72 \%$ vs. $38 \%$ and $66.6 \%$, respectively, Groups 1 and 2). Arterial hypertension $(\mathrm{AH})$ history with the same frequency was found in all comparisons, but it should be noted that the duration of AH was significantly higher in Group 3 with LV diastolic dysfunction type 3 (9.5 versus 4.9 and 6.5 years in Group 1 and 2, respectively, $P<0.05)$. Diabetes mellitus (DM) with a significant frequency prevailed in Group 3 patients $(20 \%$ vs. $7.5 \%, 7.1 \%$ in Group 1 and 2 , respectively). A similar trend can be seen in relation to CHD duration before the MI onset: 10.8 years in Group 3 with severe LV diastolic dysfunction compared with 4.1 and 6.5 years in Groups 1 and 2 , respectively $(P<0.05)$. Significant differences were also identified in relation to body mass index (BMI). BMI was 30.5 $\mathrm{kg} / \mathrm{m}^{2}$ in Group 3 vs. $27.1 \mathrm{~kg} / \mathrm{m}^{2}$ and $27.7 \mathrm{~kg} / \mathrm{m}^{2}$ in Groups 1 and 2 , respectively.

Table 2.

Comparative characteristics of groups with different types of $L V$ diastolic dysfunction

\begin{tabular}{|l|c|c|c|}
\hline \multicolumn{1}{|c|}{ Variable } & Group 1 & Group 2 & Group 3 \\
\hline Anterior MI & $31 / 38 \%$ & $28 / 66.6 \% *$ & $18 / 72 \%^{\wedge}$ \\
\hline Posterior MI & $49 / 61.2 \%$ & $14 / 33.4 \% *$ & $7 / 28 \%^{\wedge}$ \\
\hline AH & $72 / 90 \%$ & $32 / 76.2 \%$ & $20 / 80 \%$ \\
\hline DM & $6 / 7.5 \%$ & $3 / 7.1 \%$ & $3 / 20 \%{ }^{\wedge . \Delta}$ \\
\hline Aneurism & $24 / 30 \%$ & $11 / 26.2 \%$ & $13 / 52 \%$ \\
\hline Thrombolysis & $11 / 13.7 \%$ & $5 / 12 \%$ & $3 / 12 \%$ \\
\hline EPIA & $30 / 37.5 \%$ & $17 / 40.1 \%$ & $10 / 40 \%$ \\
\hline BMI, $\mathrm{kg} / \mathrm{m}^{2}$ & $27.1+3.8$ & $27.7+3.7$ & $30.5+4.9^{\wedge . \Delta}$ \\
\hline RWT & $0.40+0.10$ & $0.34+0.09$ & $0.35+0.10$ \\
\hline LVMI, g/m ${ }^{2}$ & $133.4+37.7$ & $132.3+34.5$ & $141.6+38.4$ \\
\hline IVSIdiast, $\mathrm{cm} / \mathrm{m}^{2}$ & $1.11+0.29$ & $0.97+0.27$ & $1.0472+0.37$ \\
\hline LV EDVI, $\mathrm{ml} / \mathrm{m}^{2}$ & $74.4+19.9$ & $89.9+28.7^{*}$ & $93.8+36.3^{\Delta}$ \\
\hline LV ESVI, $\mathrm{ml} / \mathrm{m}^{2}$ & $38.5+16.4$ & $48.97+20.38$ & $50.9+23.47^{\Delta}$ \\
\hline LA ESVI, $\mathrm{ml} / \mathrm{m}^{2}$ & $38.7 \pm 1.9$ & $44.8 \pm 2.6$ & $52.0 \pm 2.4^{\wedge . \Delta}$ \\
\hline LVEF, $\%$ & $49.2+11.6$ & $46.08+9.33$ & $38.4+12.06^{\wedge . \Delta}$ \\
\hline
\end{tabular}

* $-P<0.05$ between Groups 1 and 2; ${ }^{\wedge} P<0.05$ between Groups 2 and $3 ;{ }^{-}-P<0.05$ between Groups 1 and 3

Analysis of the geometry and contractility of the left chambers of the heart showed that the LV volume indicators (the indexed EDV and ESV) in patients of Groups 2 and 3 were significantly higher compared with Group 1 patients. In particular, the indexed EDV and ESV were higher by $20 \%$ and $27 \%$ in Group 2 vs. Group 1, and by $26 \%$ and $32 \%$ in Group 3 vs. Group 1, respectively $(P<0.05)[9-11]$.

Group 3 patients experienced an increase in dilatation of the left chambers and LVM, a decrease in LV contractility, and significant differences in the index of contractile function of the left atrium. The indexed ESV of the left atrium was 
significantly different compared to this parameter in Groups 1 and $2(P<0.05)$.

According to 24-hour ECG monitoring, VAs, including the potentially malignant forms, were detected in $81 / 57 \%$ and $54 / 36.7 \%$ patients, respectively, on the 12 th through the 14 th days of MI. In Q-MI patients, the comparative analysis of the structure of the ectopic activity, depending on the type of LV diastolic dysfunction, identified a high grade of VA, according to the J.Bigger classification, in $55.6 \%, 81.2 \%$ and $78.5 \%$ of cases in Groups 1, 2, and 3, respectively. A similar trend was observed in relation to PVCs, according to the Lown-Wolf classification (Table 3). In particular, Class I was detected in $11 / 50 \%, 5 / 26.3 \%$ ) and $1 / 7.7 \%$ patients in Groups 1,2 , and 3 , respectively; Class II in $4 / 18.2 \%, 2 / 21.1 \%$, and $2 / 15.4 \%$ patients in Groups 1, 2, and 3, respectively; Class III in $6 / 27.3 \%, 7 / 36.8 \%$, and $4 / 30.8 \%$ patients in Groups 1,2 , and 3 , respectively. Class IV $+\mathrm{V}$ was detected in $1 / 4.5 \%, 3 / 15.8 \%$, and $6 / 46.2 \%$ patients in Groups 1,2 , and 3, respectively. We did not observe significant differences in the registration of Class II and Class III PVCs between the groups of patients with type 2 and type $3 \mathrm{LV}$ diastolic dysfunction, but complex forms of PBCs were often identified in Group 3 patients with the restrictive type of diastolic dysfunction vs. Group 2 patients with a pseudonormal type of diastolic dysfunction $(P<0.05)$.

Table 3.

PVCs in patients with different types of LV diastolic dysfunction

\begin{tabular}{|l|c|c|c|}
\hline \multicolumn{1}{|c|}{ PVCs } & Group 1 & Group 2 & Group 3 \\
\hline $\begin{array}{l}\text { Number of patients } \\
\text { with PVCs }\end{array}$ & 22 & 19 & 13 \\
\hline Class I, n/\% & $11 / 50 \%$ & $5 / 26.3 \%$ & $1 / 7.7 \% *$ \\
\hline Class II, n/\% & $4 / 18.2 \%$ & $4 / 21.1 \%$ & $2 / 15.4 \%$ \\
\hline Class III, n/\% & $6 / 27.3 \%$ & $7 / 36.8 \%$ & $4 / 30.8 \%$ \\
\hline Class IV+V, n/\% & $1 / 4.5 \%$ & $3 / 15.8 \%$ & $6 / 46.2 \% * \wedge$ \\
\hline
\end{tabular}

$*_{-} P<0.05$ between Groups 1 and $3 ; \wedge^{\wedge}-P<0.05$ between Groups 2 and 3 .

In conclusion: The progression of LV diastolic dysfunction in Q-MI patients is associated with a longer history of CHD and hypertension. With worsening diastolic dysfunction, a marked decrease in LV systolic function is revealed. The severe diastolic dysfunction in Q-MI patients is closely associated with myocardial electrical instability.

\section{References}

1. Boldueva SA, Shabrov AV, Nesterko AO, Leonova IA, Burak TIa, Samokhvalova MV, et al. Factors affecting development of sudden death and risk stratification of patients after myocardial infarction. Kardiologiia 2006; 46(6):64-5. [Article in Russian].

2. Zipes DP, Camm AJ, Borggrefe M, Buxton AE, Chaitman B, Fromer M, Gregoratos G, et al. ACC/AHA/ESC Guidelines for Management of Patients with Ventricular Arrhythmias and the Prevention of Sudden Cardiac Death. J Am Coll Cardiology 2006; 48(5):247-346.

3. Arbolishvili GN1, Mareev VI, Orlova IA, Belenkov IN. Heart Rate Variability in Chronic Heart Failure and Its Role in Prognosis of the Disease. Kardiologiia 2006; 46(12):4-11. [Article in Russian].

4. Meta-Analysis Research Group in Echocardiography (MeRGE) AMI Collaborators, Møller JE, Whalley GA, Dini FL, Doughty RN, Gamble GD, Klein AL, et al. Independent prognostic importance of a restrictive left ventricular filling pattern after myocardial infarction: an individual patient metaanalysis: Meta-Analysis Research Group in Echocardiography acute myocardial infarction. Circulation 2008; 117(20): 2591-8. 5. Nagueh SF, Appleton CP, Gillebert TC, Marino PN, Oh JK, Smiseth OA, et al. Recommendations for the evaluation of left ventricular diastolic function by echocardiography. Eur J Echocardiogr 2009; 10(2):165-93.

6. Devereux RB, Reichek N. Echocardiographic determination of left ventricular mass in man. Anatomic validation of the method. Circulation 1977; 55(4):613-8

7. Kapel'ko VI. Diastolic dysfunction. Kardiologiia 2011; 51(1):79-90. [Article in Russian].

8. Somaratne JB, Whalley GA, Poppe KK, Gamble GD, Doughty RN. Pseudonormal mitral filling is associated with similarly poor prognosis as restrictive filling in patients with heart failure and coronary heart disease: a systematic review and meta-analysis of prospective studies. J Am Soc Echocardiogr 2009; 22(5):494-8.

9. Kindermann M, Reil JC, Pieske B, van Veldhuisen DJ, Böhm M. Heart failure with normal left ventricular ejection fraction: what is the evidence? Trends Cardiovasc Med. 2008; 18(8):280-92.

10. Sohn DW. Heart failure due to abnormal filling function of the heart. J Cardiol 2011; 57(2):148-59.

11. Andersen NH, Karlsen FM, Gerdes JC, Kaltoft A, Bøttcher M, Sloth E, et al. Diastolic dysfunction after an acute myocardial infarction in patients with antecedent hypertension. J Am. Soc. Echocardiogr 2008; 21(2):171-7. 